\title{
NORMAL-WEIGHT OBESITY FREQUENCY \\ IN THE CENTRAL EUROPEAN URBAN ADULT \\ FEMALE POPULATION OF BRNO, CZECH REPUBLIC
}

\author{
Martin Čuta ${ }^{1,2}$, Klára Bařicová1, Dominik Černý1, 2 , Ondřej Sochor ${ }^{2}$ \\ ${ }^{1}$ Department of Anthropology, Faculty of Science, Masaryk University, Brno, Czech Republic \\ ${ }^{2}$ International Clinical Research Centre, St. Anne's University Hospital Brno, Brno, Czech Republic
}

\section{SUMMARY}

Objectives: The universally recognized indicator of nutritional status, BMI, has some shortcomings, especially in detecting overweight and obesity. A relatively recently introduced normal weight obesity (NWO) describes a phenomenon when individuals are found to have normal weight as indicated by BMI but have an elevated percentage of body fat. Normal weight obese individuals face a higher risk of developing metabolic syndrome, cardiometabolic dysfunction and have higher mortality. No studies have been previously performed which would map NWO in Brno, Czech Republic.

Methods: In a sample of 100 women from Brno, we assessed the percentage of normal weight obese individuals using bioelectric impedance analysis (BIA) - three different analyzers were utilized: Tanita BC-545 personal digital scale, InBody 230 and BodyStat 1500MDD. Also, a caliperation method was used to estimate body fat percentage. Various body fat percentage cut-off points were used according to different authors.

Results: When the $30 \%$ body fat (BF) cut-off was used, up to $14 \%$ of the women in our sample were found to be normal weight obese. When the sum of skinfolds or the $35 \% \mathrm{BF}$ cut-off point are selected as a criterion for identifying normal weight obesity (NOW), only 1 of 100 examined women was identified as normal weight obese; at the $35 \%$ BF cut-off, BodyStat analyzer categorized no women as normal weight obese. Also, when the $30 \% \mathrm{BF}$ or 66 th percentile $\mathrm{BF}$ cut-off points were utilized, BodyStat identified pronouncedly fewer women from our sample to be normalweight obese than the two other analyzers.

Conclusions: On a pilot sample of Czech women, we demonstrated that depending on the selected cut-off (there is no clear agreement on cut-off points in literature), up to $14 \%$ of the examined women were found to be normal weight obese.

Key words: normal weight obesity, body fat, bioimpedance, skinfolds, risk factor of cardiovascular disease

Address for correspondence: O. Sochor, International Clinical Research Centre, St. Anne's University Hospital Brno, Pekařská 53, 65691 Brno, Czech Republic. E-mail: ondrej.sochor@fnusa.cz

https://doi.org/10.21101/cejph.a5133

\section{INTRODUCTION}

Overweight and obesity are considered a global problem affecting all strata of the human population $(1,2)$, which has led to the notion "epidemic of obesity" or, especially for children and adolescents, "pandemic of obesity" (3). The prevalence of overweight individuals in absolute numbers is staggering, as are the subsequent health risks with respective individual and population/healthcare/social costs. The percentage projections are even more disturbing: according to estimates based on 2005 population data, up to $57.8 \%$ of the population could be either overweight or obese by 2030 (4). However, the majority of these estimates were created using BMI data. With the assistance of experts who participated in the International Obesity Task Force in 1997, the World Health Organization (WHO) stated that an adult person is considered obese when $\mathrm{BMI} \geq 30 \mathrm{~kg} / \mathrm{m}^{2}(5)$. However, obesity is defined (also by the WHO) as "abnormal or excessive fat accumulation that may impair health" (6). Therefore, BMI cannot be an objective indicator of obesity as it does not provide an assessment of how individual body composition components affect overall body weight. Therefore, apart from misdiagnosing muscular athletes as obese although their elevated BMI is caused by enhanced skeletal muscle mass, individuals with low fat free mass and a high content of body fat can be found to have normal body mass index (7). BMI also cannot differentiate between central and peripheral fat (8). Central (or abdominal) obesity is strongly associated with a risk of future cardiovascular disease (9).

In addition to BMI, there are several other methods that can be used to estimate adiposity. There has been great progress made in the field of biophysical advanced methods to study body composition, yet among anthropologists, there remains a strong tendency not to abandon traditional caliperation methods (based on skinfold thicknesses measured at different numbers of standard locations and using different equations to estimate total subcutaneous fat depending on the author). There is even a wider variety of biophysical or clinical advanced methods used to estimate body fat content in humans (10). Of these methods, the method of bioelectric impedance analysis (BIA) is the most ubiquitous thanks to its relatively low-cost and easy-to-use operation. Although its reliability when compared to a reference method (DEXA) is disputed, several authors have found the method to be satisfactorily reliable (11-14). 
As mentioned above, BMI as an indicator of obesity can lead to both false-positive and false-negative results. Especially troubling is the latter case - a person whose BMI indicates normal values of weight, but is not aware that he/she has an elevated percentage of body fat (or does not realize its seriousness) might feel at ease, yet faces a higher risk of developing metabolic syndrome or cardiometabolic dysfunction and might face higher mortality (7). De Lorenzo et al. (15) first described the association between normal values of BMI, increased body fat $(\mathrm{BF})$ percentage and metabolic abnormalities and defined a new term/risk factor: normal weight obesity (NWO).

However, just as there is no clear understanding among authors as to the "cut-off" values of body fat that indicate obesity, the same is true of normal weight obesity cut-offs (7). There are various definitions of normal weight obesity - the majority of all authors agree that NWO is defined by normal value of body weight and BMI $\left(<25 \mathrm{~kg} / \mathrm{m}^{2}\right)$ and some indicator of nutritional imbalance. Some define it as an increased percentage of body fat - the authors of the original study (15) use a fixed cut-off point (>30\%). Other investigators have proposed sex- and gender-specific BF cut-offs, for women varying between 30 and 37\%; some authors propose different BF percentiles -66 th (16) up to the 95 th percentile (17). Madeira et al. (18) used an anthropometric indicator to evaluate adiposity in NWO identification - as a cut-off value, they used the 90th percentile for the sum of triceps and subscapular skinfolds. We have adopted several of the proposed NWO definitions/cutoffs to identify such women in a Czech sample.

This study aims to assess the percentage of NWO individuals in a sample of Brno women using various methods and cut-off points.

\section{MATERIALS AND METHODS}

One hundred young women aged between $20-30$ years participated in this study. The study participants were recruited among Gynfit, Ltd. gynaecological centre patients using a consecutive sampling technique. Participation was voluntary and anonymity was granted as each participant was assigned a unique code. Pregnant women were excluded from the study. In our consecutive sample, we reached a $71 \%$ response rate $(140$ women were addressed, 100 participated, average age $24.7 \pm 3.05 \mathrm{SD}$ ). None of the women who agreed to the examination later withdrew from the study. A signed informed consent was necessary for inclusion in the study. The informed consent form also included a leaflet containing instructions that needed to be followed to ensure the condition of measurement objectivity - no alcohol consumption 24 hours prior to examination, no strenuous physical exercise 12 hours prior, no ingestion of food or liquids 4 hours prior; it was also necessary to undergo the examination with an empty urinary bladder. In addition, the participants declared in the informed consent that they had no diagnosed heart defect or disease and that they were not in early stages of pregnancy (as bioimpedance examination can present a certain health risk in these cases).

All participants were examined by one female researcher in a closed examination room in their underwear at standard room temperature, during the centre opening hours. Because the participants came to the centre for their scheduled appointments with the physician, it was not possible to perform all examinations during the same (preferably morning) hours.
The participants' body height and weight were measured and recorded, and BMI was calculated based on measured values. Body height was measured in the standard anatomical position using a GPM anthropometer (DKSH, Switzerland), body weight was recorded using a Tanita BC-545 (Tanita Co., Japan) digital personal scale/BIA analyzer. Subscapular and triceps skinfold thicknesses were measured on the right side using a Best type caliper (model BEST II K-501, Trystom Czech Republic); the subscapular skinfold is located under the lower angle of the scapula, running obliquely (parallel to the medial scapular edge), the triceps skinfold is located on the dorsal surface of the upper arm on the triceps muscle, in the middle of the distance from the acromion scapulae to the olecranon ulnae. This skinfold is oriented vertically. A simple sum of these skinfolds was computed and the 90th percentile of the sum was used as the cut-off (18).

The participants' body fat percentage was then assessed using a bioimpedance analysis method (in compliance with all standard and manufacturer specified examination conditions). Three different bioimpedance analyzers were used: Tanita BC-545 personal digital scale, InBody 230 (InBody Co., Ltd., Korea) and BodyStat 1500MDD (Bodystat Limited, UK). All the analyzers were tetrapolar, dual-frequency and all used multiple electrodes (Tanita and InBody 8 electrodes, BodyStat 4 electrodes). However, each machine design required specific measurement protocols. Examinations performed using Tanita BC-545 and BodyStat 230 analyzers were performed standing on the machine's platform, holding either retractable handles (Tanita) or folding handles connected to a vertical control and display panel (InBody). Examinations using the BodyStat 1500MDD were performed in the supine position with the upper limbs not touching the trunk and the lower limbs not touching each other. Disposable adhesive electrodes were connected to the analyzer body via guide cables. The electrodes were placed on the dorsum of the right hand slightly proximally from the metacarpal heads and on the distal part of the right forearm where it meets the wrist. One of the remaining two electrodes was placed on the dorsum of the foot slightly proximally from the metatarsal heads and the last one was placed on the line connecting the inner and outer side of the ankle.

The following different cut-off points for body fat content percentage to identify NWO were utilized - 30\% (gender and age independent) (19), or fixed sex-specific for women (18), 35\% (fixed-sex specific for women) (20). We also used a gender specific cut-off defined by the 66th percentile of body fat percentage (16).

\section{RESULTS}

To identify normal weight obese individuals, we used two fixed different cut-offs $(19,20)$ and a cut-off based on the 66th percentile of body fat (16). The sum of triceps and subscapular skinfolds (90th percentile) was also used.

As seen in Table 1, when the sum of skinfolds or the $35 \% \mathrm{BF}$ cut-off point is selected as a criterion for identifying NWO, only 1 of the 100 examined women was identified as normal weight obese; at the $35 \%$ BF cut-off, the BodyStat analyzer categorized no women as normal weight obese. Also, when the $30 \% \mathrm{BF}$ or 66th percentile BF cut-off points were utilized, BodyStat identified pronouncedly fewer women from our sample to be normal weight obese than the two other analyzers. Using the sex-specific 
66th percentile of body fat in our sample, the results were similar for all three analyzers. Tanita BC-545 (the 66th BF percentile for this device was $29.4 \% \mathrm{BF}$ ) identified $13 \%$ of women as normal weight obese, InBody 230 (the 66th BF percentile for this device was $27.6 \% \mathrm{BF}$ ) identified $14 \%$ of women as normal weight obese and BodyStat 1500MDD (the 66th BF percentile for this device was $26.5 \% \mathrm{BF}$ ) identified $12 \%$ of women as normal weight obese. When sum of skinfolds (90th percentile) was used to identify normal weight obese individuals in our sample, only one of the women was found to be NWO (Table 2).

\section{DISCUSSION}

Our findings are in accordance with Marques-Vidal et al. (21), who utilized a $30 \%$ BF cut-off point (fixed sex-specific for women) on a sample of the Portuguese population. They reported a $10 \%$ prevalence of normal weight obese women. However, in our study, we used three analyzers and although generally agreeing with the above stated conclusion, individual findings were significantly different. These findings are discussed in a previous paper (10). When using a fixed sex-specific cut-off point which identifies NWO on a higher (35\%) level of BF in our sample, only one woman was found to be normal weight obese (and one of the analyzers, the BodyStat, did not identify any woman to be normal weight obese). Other authors recommend using age and sex-specific cut-off points. Kyle et al. (17) recommend as cut-off an age and sex-specific value - the 95th percentile of the corresponding population. Using this cut-off, no women in our sample were found to be normal weight obese. Marques-Vidal et al. (16) proposed a different sex-specific cut-off point for women based on the 66th percentile of body fat. Using this parameter, $12 \%$ to $14 \%$ of women from our sample (depending on the BIA device used) were classified as NWO. These values are substantially higher than what Marques-Vidal et al. (16) found $-5.4 \%$ of women with NWO in their sample.

In this paper, on a small sample of young women, we aimed to ascertain whether in Czech women the normal weight obese proportion follows the prevalence found in other European countries. We believe it is important that anthropologists and health professionals understand the concept of normal weight obesity. Our results show that based on the selected method/parameters/device and cut-off points, the frequency of NWO varies significantly,

Table 1. Percentage of women with normal weight obesity bioimpedance analysis $(N=100)$

\begin{tabular}{|l|c|c|c|}
\hline NWO as defined by & Tanita BC-545 & InBody 230 & $\begin{array}{c}\text { BodyStat } \\
\text { 1500MDD }\end{array}$ \\
\hline$\geq 35 \%$ BF & $1(1 \%)$ & $1(1 \%)$ & 0 \\
\hline$\geq 30 \%$ BF & $13(13 \%)$ & $12(12 \%)$ & $5(5 \%)$ \\
\hline 66 th percentile BF & $13(13 \%)$ & $14(14 \%)$ & $12(12 \%)$ \\
\hline
\end{tabular}

Table 2. Percentage of women with normal weight obesity caliperation method $(N=100)$

\begin{tabular}{|l|c|}
\hline $\begin{array}{l}\text { Normal weight obesity as } \\
\text { defined by }\end{array}$ & $\begin{array}{c}\text { Sum of triceps and subscapular } \\
\text { skinfolds }\end{array}$ \\
\hline 90th percentile sum of skinfolds & $1(1 \%)$ \\
\hline
\end{tabular}

but up to $14 \%$ of women can be misidentified as metabolically healthy with a low cardiovascular risk. This false-negative identification bears a high risk - several authors found an association of NWO with metabolic dysregulation, inflammation and metabolic syndrome - all risk factors of coronary heart disease and cardiovascular disease $(8,15,18,19)$. In addition, Romero-Corral et al. (8) demonstrated that NWO is associated with cardiovascular disease and all-cause mortality. Even more, it was shown that normal weight central obesity defined by the waist-hip ratio is associated with higher mortality than BMI-defined obesity (22). In a subsample of women included in NHANES study, women with normal weight central obesity also had a higher mortality risk than those with a similar BMI, but no central obesity, as well as those who were obese according to BMI only (22). However, as mentioned above, there is no clear understanding on defining normal weight obesity (and obesity in general) by body fat content. Body fat distribution is also an important cardiovascular disease risk factor (23). For these reasons, the information about determining NWO in the general population is limited and further studies are needed to shed light on the relation between body fat content (and finding optimal cut-offs) and its distribution and metabolism, cardiovascular disease and mortality.

\section{Limitations of the Study}

The study was conceived as an introductory survey of NWO occurrence in the city of Brno. When evaluating obesity, indicators of thyroid function, fasting glycaemia, information on the use of contraceptives form a valuable background. The pilot nature of the study and its extent did not allow for collection of this data and fasting glycaemia, thyroid function indicators, contraceptive use etc. are not available. The fact that our sample only includes young women can be considered a limiting factor as only one sex is monitored.

\section{CONCLUSION}

BMI has been widely disputed as an indicator of obesity. Various substituting methods have been proposed, and quite recently, the concept of normal weight obesity was introduced by De Lorenzo et al. (15). On a relatively small sample of Czech women, we demonstrated that depending on the selected cut-off, up to $14 \%$ of the women were found to be normal weight obese. NWO is strongly associated with cardiovascular disease. This high-risk group needs to be recognized in clinical practice as well as in research for better adiposity-based risk stratification and prevention/treatment planning.

\section{Acknowledgements}

This study was supported by project No. LQ1605 from the National Program of Sustainability II (MEYS CR) and by the FNUSA-ICRC No. CZ.1.05/1.1.00/02.0123 (OP RDI) project.

\section{REFERENCES}

1. Lobstein T, Baur L, Uauy R; IASO International Obesity TaskForce. Obesity in children and young people: a crisis in public health. Obes Rev. 2004 May;5 Suppl 1:4-104. 
2. Finucane MM, Stevens GA, Cowan MJ, Danaei G, Lin JK, Paciorek CJ, et al.; Global Burden of Metabolic Risk Factors of Chronic Diseases Collaborating Group (Body Mass Index). National, regional, and global trends in body-mass index since 1980: systematic analysis of health examination surveys and epidemiological studies with 960 country-years and 9·1 million participants. Lancet. 2011 Feb 12;377(9765):557-67.

3. Larson IN, Story M. The pandemic of obesity among children and adolescents: What actions are needed to reverse current trends? J Adolesc Health. 2007;41(6):521-2.

4. Kelly T, Yang W, Chen CS, Reynolds K, He J. Global burden of obesity in 2005 and projections to 2030. Int J Obes (Lond). 2008 Sep;32(9):1431-7.

5. Obesity: preventing and managing the global epidemic. Report of a WHO consultation. World Health Organ Tech Rep Ser. 2000;894:1-253.

6. World Health Organization. Obesity and overweight Fact sheet $\mathrm{N}^{\circ} 311$ [Internet]. Geneva: WHO; 2015 [cited 2016 March 17]. Available from: http://www.who.int/mediacentre/factsheets/fs311/en

7. Oliveros E, Somers VK, Sochor O, Goel K, Lopez-Jimenez F. The concept of normal weight obesity. Prog Cardiovasc Dis. 2014 JanFeb;56(4):426-33.

8. Romero-Corral A, Somers VK, Sierra-Johnson J, Korenfeld Y, Boarin S, Korinek J, et al. Normal weight obesity: a risk factor for cardiometabolic dysregulation and cardiovascular mortality. Eur Heart J. 2010;31(6):73746.

9. Lee CM, Huxley RR, Wildman RP, Woodward M. Indices of abdominal obesity are better discriminators of cardiovascular risk factors than BMI: a meta-analysis. J Clin Epidemiol. 2008;61(7):646-53.

10. Čuta M, Bařicová K. Comparison of body fat percentage estimation methods in young women from Brno and its surroundings. Ceska Antropol. 2016;66(2):4-9. (In Czech.)

11. Karelis AD, Chamberland G, Aubertin-Leheudre M, Duval C; Ecological mobility in Aging and Parkinson (EMAP) group. Validation of a portable bioelectrical impedance analyzer for the assessment of body composition. Appl Physiol Nutr Metab. 2013 Jan;38(1):27-32.

12. Gupta N, Balasekaran G, Govindaswamy VV, Hwa CY, Shun LM. Comparison of body composition with bioelectric impedance (BIA) and dual energy X-ray absorptiometry (DEXA) among Singapore Chinese. J Sci Med Sport. 2011;14(1):33-5.

13. Thomson R, Brinkworth GD, Buckley JD, Noakes M, Clifton PM. Good agreement between bioelectrical impedance and dual-energy X-ray absorptiometry for estimating changes in body composition during weight loss in overweight young women. Clin Nutr. 2007;26(6):771-7.
14. Andreoli A, Melchiorri G, De Lorenzo A, Caruso I, Sinibaldi Salimei P, Guerrisi M. Bioelectrical impedance measures in different position and vs dual-energy X-ray absorptiometry (DXA). J Sports Med Phys Fitness. 2002 Jan;42(2):186-9.

15. De Lorenzo A, Del Gobbo V, Premrov MG, Bigioni M, Galvano F, Di Renzo L. Normal-weight obese syndrome: early inflammation? Am J Clin Nutr. 2007;85(1):40-5.

16. Marques-Vidal P, Pécoud A, Hayoz D, Peccaud F, Mooser V, Waeber G, et al. Normal weight obesity: relationship with lipids, glycaemic status, liver enzymes and inflammation. Nutr Metab Cardiovasc Dis. 2010 Nov;20(9):669-75.

17. Kyle UG, Genton L, Slosman DO, Pichard C. Fat-free and fat mass percentiles in 5225 healthy subjects aged 15 to 98 years. Nutrition. 2001;17(7-8):534-41.

18. Madeira FB, Silva AA, Veloso HF, Goldani MZ, Kac G, Cardoso VC, et al. Normal weight obesity is associated with metabolic syndrome and insulin resistance in young adults from a middle-income country. PLoS One. 2013;8(3):e60673. doi: 0.1371/journal.pone.0060673.

19. Di Renzo L, Galvano F, Orlandi C, Bianchi A, Di Giacomo C, La Fauci L, et al. Oxidative stress in normal-weight obese syndrome. Obesity (Silver Spring). 2010 Nov;18(11):2125-30.

20. Lavie CJ, Milani RV, Ventura HO, Romero-Corral A. Body composition and heart failure prevalence and prognosis: getting to the fat of the matter in the "obesity paradox." Mayo Clin Proc. 2010 Jul;85(7):605-8.

21. Marques-Vidal P, Chiolero A, Paccaud F. Large differences in the prevalence of normal weight obesity using various cut-offs for excess body fat. E Spen Eur E J Clin Nutr Metab. 2008;3(4):e159-62. doi:10.1016/j. eclnm.2008.05.003.

22. Sahakyan KR, Somers VK, Rodriguez-Escudero JP, Hodge DO, Carter RE, Sochor O, et al. Normal-weight central obesity: implications for total and cardiovascular mortality. Ann Intern Med. 2015;163(11):827-35.

23. Mathieu P, Poirier P, Pibarot P, Lemieux I, Despres JP. Visceral obesity: the link among inflammation, hypertension, and cardiovascular disease. Hypertension. 2009;53(4):577-84. 